$\underline{\text { Research article }}$

\title{
Clinico-pathological correlation and outcome analysis of disseminated histoplasmosis treated with conventional Amphotericin B.
}

\author{
D Modak $^{1,} \mathrm{~N}_{\text {Dutta }}{ }^{1}$, Rajyasree De ${ }^{1}$, SK Guha ${ }^{1}$ \\ Sri Lankan Journal of Infectious Diseases 2015 Vol.5 (2):86-91 \\ DOI: http://dx.doi.org/10.4038/sljid.v5i2.8080
}

\begin{abstract}
Introduction

Disseminated histoplasmosis is a treatable common opportunistic infection in HIV infected people and not uncommon in others in a tropical country like India. The objective of our study was to evaluate clinical-pathological correlation and treatment outcome of disseminated histoplasmosis treated with conventional Amphotericin B in an endemic area.
\end{abstract}

\section{Material and methods}

This was a retrospective observational study of twenty-two cases of disseminated histoplasmosis admitted to a tertiary care hospital from January 2009 to December 2012 and treated with Amphotericin B followed by oral Itraconazole therapy for one year. Results of treatment outcome including relapse and mortality were analyzed in January 2014.

\section{Results}

Histoplasmosis was diagnosed in patients with advanced HIV (72\%) illness with a mean CD4 count of 63.43/1. Tuberculosis and diabetes were other co-morbid illnesses and it was less common among immunocompetent patients (9\%). Fifty percent of the patients presented with cutaneous lesions along with systemic manifestations while $27 \%$ had only mucocutaneous lesions. Adrenal histoplasmosis (18\%) was common in HIV negative subjects. HIV positive patients showed excellent response to Amphotericin B followed by Itraconazole therapy. In 27\% HIV positive patients, the disease manifested as IRIS (immune reconstitution inflammatory syndrome). Relapse was seen in $2(9 \%)$ patients. After one year of completion of therapy 16 patients were cured, 3 patients $(13.6 \%)$ died in the early part of treatment and one was lost to follow up.Treatment response in HIV infected patients showed excellent results but long term maintenance itraconazole therapy was inevitable (12-32 months).

\section{Conclusions}

It is concluded that early diagnosis and treatment can prevent a fatal disease like disseminated histoplasmosis with conventional Amphotericin B followed by Itraconazole. Adrenal histoplasmosis was common among the HIV negative population. Extensive follow up is required to identify early relapse which may need further prolongation of therapy for cure.

Key words: Histoplasmosis, HIV, immunocompetent, Amphotericin B.

${ }^{1}$ Centre of excellence in HIV care, School of Tropical Medicine, Kolkata , India

Address for correspondence: Dolanchampa Modak, 4/1,Raj Kumar Chatterjee Road, Kolkata,700037,West Bengal, India, email-drdolanmodak@gmail.com, tele : 919433118424.

Received 14 April 2015 and revised version accepted 14 October 2015 


\section{Introduction}

Histoplasma capsulatum is a dimorphic fungus, responsible for the commonest cause of systemic mycosis. ${ }^{1}$ The organism is endemic in the Ohio and Mississippi river basin areas of the United States and is also reported from parts of Central and South America, Europe, Africa and Asia. Histoplasmosis is an infrequently reported disease in India and only sporadic cases from different regions of this country have been reported in the literature. ${ }^{2}$ Infection is caused by inhalation of microspores and contaminated soil which remains potentially infectious for many years and is most often found near bat and bird habitats. ${ }^{3}$ This organism is of low human virulence and only clinically manifests when host immune responses allow persistent parasitisation of macrophages. ${ }^{4}$ Disseminated histoplasmosis (DH) runs a varied clinical course ranging from asymptomatic selfhealing illness, to acute or chronic pulmonary disease and an acute to chronic progressive form, depending upon the immune status of the host, virulence of the organism and size of inoculum. Acute progressive disseminated illness, which presents with abrupt onset of fever, organomegaly, lymphadenopathy and cytopenia without any granulomatous response is usually fatal. The subacute variety follows a relentless course with focal lesions and organomegaly. The chronic form has an indolent course with focal lesions, organomegalies and an effective cell mediated immune response. Its clinical features overlap with those of other systemic illnesses of tropical countries such as tuberculosis, leishmaniasis, sarcoidosis and malignancies. Diagnosis therefore requires a high index of suspicion with recognition of common modes of presentation and familiarization with diagnostic tests.

The aim of our study was to analyze treatment responsiveness, outcome and mortality in terms of age, sex, type of presentation, associated illness, CD4 cell count, clinical manifestations, laboratory and radiographic investigations in diagnosed cases of disseminated histoplasmosis (DH) in a tertiary care hospital.

\section{Materials and methods}

We retrospectively analyzed the recorded data of patients diagnosed with DH admitted to Carmichael Hospital for Tropical Diseases, a tertiary referral center in Eastern India, from January 2009 to December 2012 and followed up till December 2013.We defined DH using the following criteria.

a) The presence of extra pulmonary H.capsulatum with or without granuloma seen using the Periodic acid-Schiff (PAS) or Grocott's methenamine silver (GMS) stain from aspirated material and biopsy specimens (skin, oral lesion and palatal mucosa, adrenal gland).

b) Positive culture (tissue/bone marrow) in Sabouraud dextrose agar media. In some patients the organism was isolated from more than one site.

HIV was diagnosed by standard ELISA method according to NACO protocol.

We defined acute illness if symptoms were present for 2 weeks or less, sub-acute between 2 to 6 weeks of symptoms and chronic if more than 6 weeks of symptoms. Routine laboratory investigation records including complete haemogram, fasting and post prandial blood sugar, liver function tests, urea, creatininine, LDH records were monitored. Imaging studies including chest Xray, Ultrasonography of abdomen, CECT abdomen (if needed) were done. In HIV positive patients CD4 count and combination anti- antiretroviral therapy (cART) records were monitored from AntiRetroviral Therapy (ART) Centre. Institutional ethical clearance was obtained. 


\section{Results and Analysis}

In our study twenty-two patients were diagnosed as DH (Male: Female ratio was 19:3). DH was diagnosed by demonstration of organism from Fine-needle aspiration cytology (FNAC) and/or

Table 1 : Diagnostic samples

\begin{tabular}{lcc}
\hline Specimen & No of patients & \% \\
\hline FNAC/biopsy & 19 & 86 \\
Culture (from lesions) & 4 & 18 \\
Bone marrow culture & 3 & 13 \\
\hline
\end{tabular}

biopsy material in 19 patients, fungal culture from aspirated material of cutaneous lesions in 4 and bone marrow in 3 patients. Four patients were diagnosed by cytology /biopsy using High-dose contrast-enhanced computed tomography (CECT) guided FNAC of the adrenal gland (Table 1). Sixteen patients had HIV infection, 4 were diabetics, 5 had pulmonary tuberculosis and 8 patients had extra pulmonary tuberculosis. Two patients were immunocompetent and had no co-morbid conditions (Table 2). Average age at presentation was

Table 2

Associated comorbidities and outcomes in patients with disseminated histoplasmosis

\begin{tabular}{lcc}
\hline & No & $\%$ \\
\hline \multicolumn{2}{c}{ Associated comorbidity } \\
\hline HIV positive & 16 & 72.7 \\
(HIV negative & 6 & $27.3)$ \\
Diabetes mellitus & 4 & 18.2 \\
Pulmonary TB & 5 & 22.7 \\
Extra-pulmonaryTB & 8 & 36.4 \\
\hline Immunocompetent patients & 2 & 9.1 \\
\hline \multicolumn{3}{c}{ Patient outcomes } \\
\hline Cure & 16 & 72.7 \\
Recurrence & 2 & 9.1 \\
Death & 3 & 13.6 \\
Lost to follow up & 1 & 4.5 \\
\hline
\end{tabular}

Table 3 : Clinical features in patients with disseminated histoplasmosis

\begin{tabular}{lcc}
\hline \multicolumn{1}{c}{ Symptom } & No & \% \\
\hline Fever & 15 & 68.2 \\
Weakness & 16 & 72.7 \\
Weight loss & 6 & 27.3 \\
Abdominal pain & 1 & 4.5 \\
Papulo-nodular skin lesions & 11 & 50 \\
Oral / mucosal ulcers & 6 & 27.3 \\
Hepatosplenomegaly & 7 & 31.8 \\
Splenomegaly & 2 & 9.1 \\
Lymphadenopathy & 4 & 18.2 \\
\hline
\end{tabular}

36.7 years, (range 13-58). All patients were from the gangetic belt of West Bengal except 1 patient who was from Orissa.

Among the HIV positive patients, the mean CD4 count was $63.43 / \mu 1$ (range 16-152) at the time of diagnosis of DH. Fifteen $(68.18 \%)$ patients complained of fever, 16 (72\%) had weakness, 6 $(27.27 \%)$ had weight loss and one patient $(4.5 \%)$ complained of abdominal pain.

On examination, few non- tender papulo-nodular, crusted skin lesions were present on the face in $50 \%$ of patients with oral and mucosal ulcers in 6 $(27.2 \%)$ patients. There was hepatosplenomegaly in $7(31.8 \%)$ patients, splenomegaly in $2(9.1 \%)$ patients and lymphadenopathy in 4 (18.1\%) patients (Table 3). DH was diagnosed in $10 \mathrm{HIV}$ positive patients before the commencement of ART .In 6 patients; the disease manifested after commencement of ART and was documented as IRIS. Anemia and leucopenia was found in 21 patients and raised LDH were seen in 12 patients.

The total treatment duration with amphotericin B followed by oral itraconazole for non HIV patients was one year. However, in the HIV infected patient group, 2 died in the 1 st week after initiation of treatment. As this patient died within the first week of diagnosis, we could not complete the induction phase of Amphotericin B treatment. The third patient died just after completion of the course of Amphotericin B injection and had very low base line CD4 count

$(<100 / \mu \mathrm{l})$ before initiation of treatment. Clinical well-being was seen in thirteen patients from the 2nd week of treatment with gradual disappearance of skin lesions. Ten patients with base line CD4 count $<100 / \mu 1$ required prolonged itraconazole maintenance therapy with 4 patients 
Table 4 CD4 count in HIV positive patients

\begin{tabular}{lccccc}
\hline Time & \multicolumn{2}{c}{ Base line CD4 count } & \multicolumn{2}{c}{ CD4 count after 1.5 years } & CD4 count after 2.5 years \\
\hline CD4 count & $<100$ cells $/ \mu 1$ & $100-200$ cells $/ \mu 1$ & $<100$ cells $/ \mu 1$ & $>150$ cells $/ \mu 1$ & $>150$ cells $/ \mu 1$ \\
No of patients & 13 & 3 & 5 & 8 & 13 \\
\hline
\end{tabular}

needing 12-16 months, 5 patients 16-24 months, and one patient 32 months of treatment. One patient was lost to follow up at the 14th month .Two patients with baseline CD4 count 100200cells/ $\mu \mathrm{l}$, were treated for 12-14 month till their CD4 count was raised and maintained a steady value of $>150 / \mu$ l for 6 months (Table 4 ) .

Four patients (with diabetes and tuberculosis) were treated with amphotericin B and itraconazole for one year. Minor side effects of amphotericin B were experienced by all of them. One diabetic patient developed hypokalemia and nephrotoxicity (raised creatininine) which were managed conservatively. Two immunocompetent patients with no comorbidities were treated for twelve months. In two immunocompetent patients, disappearance of cutaneous lesions was seen after 3 months of initiation of therapy although a total one year of therapy was given to all patients. Two patients, who had features of Addison's disease, were successfullytreated for one year with Itraconazole, a corticosteroid and minerelo-corticosteroid supplement.

Relapse occurred in 2 patients which were treated with oral itraconazole for one more year. Disease was reactivated (by reappearance of clinical symptoms and laboratory confirmation) in one of them 1.5 years after completion of therapy.

HIV positive patients were treated with combination antiretroviral drugs according to NACO guideline. Diabetes and tuberculosis were managed simultaneously along with histoplasmosis.

\section{Discussion}

This was one of the few large studies conducted in India where previous studies was done sporadically. ${ }^{5,}$ In our study, HIV was the commonest associated illness (72.7\%) although with low prevalence, representing $0.85 \%$ of all admitted HIV patients. A previous study done by Mc Kinsey DS et al in 1989 showed a high prevalence rate (5-32\%) of histoplasmosis among HIV positive patients. ${ }^{7}$ The low prevalence in our study is probably due to late diagnosis and underdiagnosis in bone marrow culture. ${ }^{8}$ We diagnosed DH from FNAC (86\%), culture of tissue samples (18\%) and only $13 \%$ of patients had positive bone marrow cultures. Carlos V Paya et al in 1984 reported $46 \%$ of positive results were obtained by tissue staining and $71 \%$ positive blood cultures for $H$ capsulatum ${ }^{8}$.

Body weakness, fever and weight loss were the commonest symptoms in the study group. Similar to previous studies in other countries, $50 \%$ of our patients presented with cutaneous manifestations. ${ }^{9}$ We found that only $2(9 \%)$ immunocompetent and $27 \%$ of the entire study group had oro-mucosal lesions. M.Harnalikaret al (2012) also reported similar finding. ${ }^{10}$ SoujaFilho FJ et al in a previous report in 1995 showed DH is rare in immunocompetent persons and is usually associated with the severe disseminated form of histoplasmosis. ${ }^{11}$

Though adrenal gland histoplasmosis is a part of disseminated infection, none of the HIV positive patients showed significant clinical and radiological adrenal enlargement. Adrenal involvement was noted in 4 HIV seronegative patients. Addison's disease developed in 2 patients. Adrenal involvement in histoplasmosis resulting in adrenal insufficiency is an uncommon presentation, 
with none showing clinical evidence of adrenal failure. ${ }^{12}$ Addison's disease typically occurs with extensive destruction of both adrenal glands during infection by the organism. In early stages of destruction, it typically presents as chronic fatigue syndrome. Angeli A et al (1991) has shown that adrenal glands were enlarged and there were signs of granulomatous inflammation within the adrenals. ${ }^{13}$ Adrenal function recovers following cure with antifungal therapy unlike that seen with treatment of tuberculosis, where lifelong cortisol replacement is needed. ${ }^{6}$ Duration of treatment for adrenal histoplasmosis is not clear from existing information and treatment regimens advocate antifungals given for periods ranging from 6 months to 2 years. ${ }^{14}$ Deepak Kothari et al (2013) reported persistence of $H$. capsulatum in adrenal biopsy material 7 years after treatment with Itraconazole for nine months and suggested that prolonged therapy with regular reviews of adrenal morphology and histology is needed. ${ }^{14}$ We too have seen one of our patients with adrenal histoplasmosis relapsed after one year of completion of treatment. Another immunocompetent patient developed signs and symptoms of relapse 1.5 years after of completion of therapy.

Similar to McKinsey D S et al (1997), we have seen that very low CD4 counts (mean CD4 value $63 / \mu \mathrm{L}$ ) along with endemicity may be a possible risk factors for DH in advanced HIV illness. ${ }^{15}$ Recent studies show that for DH patients with moderately severe or severe manifestations, liposomal Amphotericin B is more effective and less toxic than the deoxycholate formulation of Amphotericin B. Patients in the study were treated with low cost deoxycholate formulation of Amphotericin B for 10 days (hospital supply) followed by Itraconazole therapy for one year (according to IDSA guidelines). Since ART was introduced, the clinical and immunologic status of HIV-infected patients has dramatically improved. We have also found that cure rate was high among treated HIV infected patients and treatment was discontinued when CD4 counts steadily rose above 150/ $\mu \mathrm{l}$. Mitchell Goldman et al (2004) showed that discontinuation of antifungal maintenance therapy appeared to be safe in HIV infected patients who achieved adequate immunological response to antiretroviral therapy $(\mathrm{CD} 4>150 / \mu \mathrm{l}){ }^{16} \mathrm{DH}$ manifested as IRIS in 6 (27\%) HIV patients in the current study. DH manifested as IRIS within two months of ART initiation and IRIS was documented by raised CD4 count and presence of granuloma. Breton et al (2006) also demonstrated IRIS in HIV positive population. ${ }^{16}$

Acute progressive DH in AIDS has a high mortality rate. Three patients presented with very advanced illness of acute variety and died in the early weeks of diagnosis before completing the induction phase of therapy with Amphotericin B. Relapse of disease was seen in 2 of our patients who required prolonged Itraconazole therapy. Prolonged suppression (secondary prophylaxis) with once-daily Itraconazole is recommended for patients who experience relapse or are in irreversible immunosuppression. ${ }^{18}$

\section{Conclusion}

Treatment with liposomal Amphotericin B is known to be superior in term of safety, toxicity and mortality for treatment of disseminated histoplasmosis. However conventional Amphotericin B is also highly effective both in immunocompromised and immunocompetent patients and can be used for its management in resource poor settings including India.

\section{Limitations}

As a tertiary hospital, moderate to severe cases are referred from different parts of Eastern India, the exact numbers of infected population particularly in endemic areas could not be assessed. We could not estimate the serum or urinary histoplasma antigen level due to the unavailability of the test. 


\section{Acknowledgement}

We are grateful to our institution, our senior and junior colleagues of tropical medicine, dermatology and microbiology department for their constant guidance. We are thankful to our patients, as without their support this study was not possible.

\section{References}

1. Wheat LJ, Chetchotisakd P, Williams B, et al. Factors associated with severe manifestation of histoplasmosis in AIDS. Clin Infect Dis 2000; 30(6):877-81. doi: http://dx.doi.org/10.1086/313824

2. Keyur S. Vyas, Robert W. Bradsher, Jr. Histoplasmosis can be severe for HIV-infected persons in endemic areas. HIV Clinician, 2011; 23:(2)1-3. No doi.

3. Chang RC, Susanto I. Histoplasmosis. $E$ medicine available from http://www.emedicine.com/med/TOPIC1021. Accessed 15 June 2009

4. Chadi A Hage, L Joseph Wheat. Infectious diseases. In: Longo, Fauci, Kasper, Hauser, Jameson, Loscalzo (eds).Harrison's principles of internal medicine, 18th edition, McGraw Hill Inc., New York, Vol 1, 2012 chapter199 pp.1640-43.

5. Randhawa HS, Khan ZU. Histoplasmosis in India: Current Status. Indian J Chest Dis Allied Sci 1994; 36:193-213. No doi.

6. S Subramanian, OC Abraham, Priscilla Rupali, et al. Disseminated histoplasmosis. JAPI 2005; 53:185-189. No doi.

7. Mc Kinsey DS, Gupta MR, Riddler SA, et al. Long term Amphotericin B therapy for disseminated histoplasmosis in patients with acquired immunodeficiency syndrome. Ann Med 1989; 111: 655659. No doi.

8. Carlos V Paya, Glenn D Roberts, Franklin R, Cockerill. Laboratory methods for diagnosis of histoplasmosis: Clinical importance of Lysis-Centrifugation blood culture technique. Mayo Clin Proc 1984; 62:480-85. No doi.

9. Rocha MM, Severo LC. Disseminated histoplasmosis in patients with acquired immunodeficiency syndrome (AIDS). Study of 25 cases. Rev Inst Med Trop Sao Paulo 1994; 36:167-70. [PUBMED]

10. Manoj Harnalikar, Vidya Kharkar, Uday Khopkar. Disseminated cutaneous histoplasmosis in an immunocompetent adult.

Indian Journal of Dermatology 2012; 57(3):206-209. No doi.

11. Souza Filho FJ, Lopen M, Almeida OP, Scully C. Mucocutaneous histoplasmosis in AIDS. $\mathrm{Br} J$ Dermatol 1995; 33:472-4. No doi.

12. Kauffman CA. Fungal infections in older adults. Clin Infect Dis 2001; 33:550-555. doi: http://dx.doi.org/10.1086/322685

13. Angeli A, Masera RG, Gatti G. Mycoses and adrenocortical function. New pathogenetic aspects of adrenal hypofunction. Recenti Prog Med 1991; 82:652-60. No doi.

14. Deepak Kothari, Shweta Chopra, Minakshi Bhardwaj, et al. Persistence of histoplasma in adrenals 7 years after antifungal therapy. Indian Journal of Endocrinology and Metabolism 2013; 17(3):529531. doi: http://dx.doi.org/10.4103/2230-8210.111679

15. McKinsey D S, Speigel R A, Hutwagner L, et al. Prospective study of histoplasmosis in patients infected with Human Immunodeficiency virus: Incidence, risk factors and pathophysiology. Clin Infect Dis 1997; 24(6):1195-1203. doi: http://dx.doi.org/10.1086/513653

16. Goldman M, Zackin R, Fichtenbaum CJ, et al. Safety of discontinuation of maintenance therapy for disseminated histoplasmosis after immununologic response to antiretroviral therapy. Clin Infect Dis 2004; 38:1485-9 doi : http://dx.doi.org/10.1086/420749

17. Breton G, Guillaume H, Adle-Biassette, et al.Immune reconstitution inflammatory syndrome in HIV-infected patients with disseminated histoplasmosis. AIDS 2006; 20(1):119-121. doi : http://dx.doi.org/10.1097/01.aids.0000199014.66139.39

18. http://www.consultantlive.com/articles/update-therapy-histoplasmosis\#sthash.lQUZ3RDI.dpuf 